\title{
SMART TEXTILES AND THEIR APPLICATIONS - VISUAL PERCEPTIONS
}

\author{
Vladan Koncar \\ University of Lille, ENSAIT - GEMTEX, Roubaix, France
}

\begin{abstract}
Smart textiles, encompassing electronics combined with textiles also called textronics, have a very promising realm in science and technology nowadays because of commercial viability and public interests. Smart textiles play a significant role as well in the European textile sector and assist the textile industry in its transformation into a competitive knowledge driven industry. These kinds of textiles combine knowledge from many disciplines with the specific requirements of textile. Numerous materials and systems are available together with devices for sensing and actuation, but they are not compatible with a textile or with the textile production processes. They could be transformed into a textile compatible structure or even in a full textile structure.

Smart textiles can be defined as textiles that are able to sense and respond to changes in their environment. They may be divided into two classes: passive and active smart textiles. Passive smart textiles have the ability to change their properties according to an environmental stimulation. Shape memory materials, hydrophobic or hydrophilic textiles etc. make part of this category.

Active smart textiles are fitted with sensors and actuators, in order to connect internal parameters to the transmitted message. They are able to detect different signals from the environment (temperature, light intensity, pollution...), to decide how to react and finally to act using various textile based, flexible or miniaturized actuators (textile displays, micro vibrating devices, LED, OLED...). The "decision" can be taken locally in case of embedded electronic devices (textile electronics) to smart textile structures or remotely in case the smart textile is wirelessly connected to clouds containing data base, servers with artificial intelligence software etc. and may be a part of Internet of Things (IOT) concept. This presentation focuses on smart textiles actuators used as active and passive flexible displays.
\end{abstract}

Key words: actuators, textile displays, smart textiles

\section{INTRODUCTION}

Two different approaches relative to the development of textile flexible displays are presented in this study. The first one, also called active display, is based ion the weaving of optical fibers and the second one (passive displays) uses electrochromic compounds within textile structures.

Upon the first approach, flexible displays can be created on textiles by producing a screen matrix using the texture of the fabric during the weaving process. A small electronic device that is integrated into the system controls the Light Emitting Diodes (LEDs) or even Diode lasers that illuminate groups of fibers. Each group provides light to one pixel on the matrix.

These displays are very thin and ultra lightweight-two characteristics that could enable many innovative applications. Although initially developed for clothing, the displays could be used to exhibit information or designs in cars, portable electronic devices and even houses and buildings. Indeed, research on the design and development of flexible displays based on processed optical fibers has opened up new frontiers in fashion, public safety, automotive equipment and home decoration.

The second approach involves materials that can undergo a reversible color change, upon the application of an external stimulus, are technically known as chromic materials. Research into the application of such species has been extensive due to the ability of color change to effectively convey important information, i.e. traffic lights, advertisement billboards etc. (Mortimer et al, 2006; Somani et al, 2003; Tehrani et al, 2006). Materials that display chromic properties are classified by the type of stimuli that induces their color change. For example, photochromics and thermochromics see a change in color when there is a change in light, or a change in heat, respectively. Both thermo and photochromic materials have current applications within textile fields. Photochromics, when applied to textiles have been used in solar protection through the monitoring and notification of UV radiation levels (Shanmugasundaram, 2008). In addition, for added interest within fashion and interior furnishings, wallpapers treated with thermochromics, when heated by nearby radiators, 'grow' flower buds across a vine (Sierra, 2008). Photochromic products include pillow covers which reveal a motif when exposed to sunlight. When the heat or sunlight is removed, the flowers and motif disappears correspondingly. 
On the other hand, an electrochromic compound is one in which a reversible change in color occurs when an electrical voltage is applied. Many materials express electrochromic properties, including metal oxides, the Prussian family, rare earth phthalocyanines and conducting polymer systems. Products that harness the fascinating spectroelectrochemical properties of electrochromic materials, currently available on the market, are predominantly rigid in their form and include the glass windows of buildings and cars which can darken reversibly (Sage Electrohromics, 2012; Gentex Corporation ,2012). Other proposed applications include re-usable price labels, camouflage materials and controllable light reflective or light-transmissive displays for optical information and storage (Mortimer et al, 2006), (Rosseinsky et al, 2001; Silver, 1989). In this study, 5 generations of electrochromic displays realized in GEMTEX laboratory are presented.

\section{FLEXIBLE TEXTILE DISPLAYS}

\subsection{Active textile displays based on optical fibers weaving}

\section{Weaving Optical Fibers}

Poly(methyl methacrylate) (PMMA) optical fibers possess a rigidity and fragility that make them different from most traditional textile fiber threads and filaments. With regard to section diameter, a good compromise must be reached: A diameter that is too large can cause inflexibility, while a too-small diameter induces a low shear resistance and loss of light intensity.

We used fibers with a diameter of $0.5 \mathrm{~mm}$ to make the first prototypes. We have also conducted tests on fibers with a diameter of $0.25 \mathrm{~mm}$, but further developments in the process of weaving are still required to ensure sufficient fabric resistance in bending.

Weaving takes place on a traditional two-dimensional loom. The optical fibers can be woven or placed as warp or weft yarns, in addition to other kinds of yarns. Therefore, it is theoretically possible to obtain an optical fiber $X-Y$ network. However, this would present several disadvantages:

- $\quad$ The grid (and, hence, the resolution) would not be very dense and the fabric would be extremely rigid because of the relatively high radius of curvature of optical fibers.

- Constituting an optical fiber chain is very long and very expensive.

- The resolution would be tiny.

It is also possible that a three-dimensional structure in weaving would not bring any significant advantages. Thus, our initial plan was to develop a fabric comprising optical fibers for wefts and polyester in warp. Other natural, artificial or synthetic yarns could also have been used to constitute the warp yarns. The choice of warp yarns must be chosen with the aim of achieving good flexibility in the fabric, fine titration and an improved capacity to diffuse and reflect the light emitted by optical fibers for better legibility of information. An example of an optical fiber fabric display (OFFD) weaved structure is shown in Figure 1. Different textile finishing methods are being tested-either in pasting or in coating - to guarantee grid stability and flame resistance and to enable optimal light emission intensity and contrast.

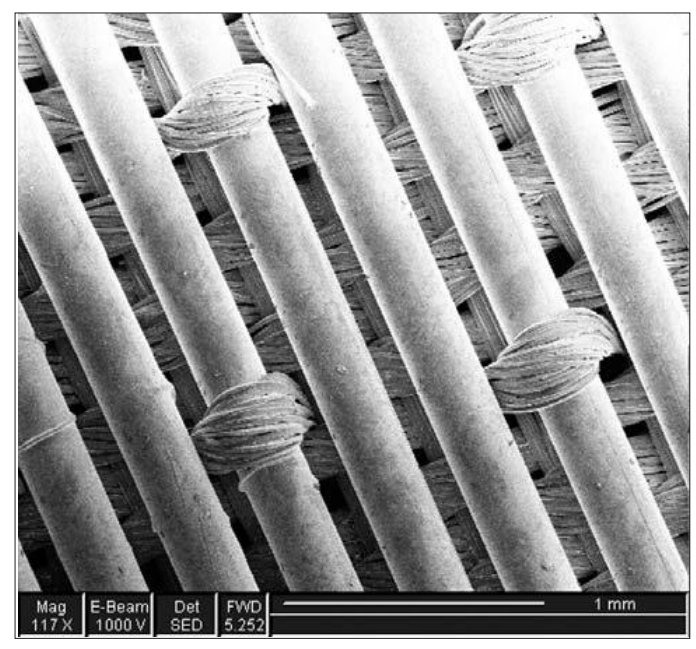

Figure 1: Scanning electron microscope picture of OFFD structure (a two-layer basic-velour fabric) 


\subsection{Display Matrix Design}

The screen for fabric displays comprises a number of surface units, or pixels; each one can be illuminated by a light source emitted from one side of the fabric by one or several PMMA optical fibers with discrete index variation. The pixels are directly formed on optical fibers while transversely forming a spout of light on the fabric. The process consists of generating micro-perforations that reach into the core of the fiber (Fig. 2). The remainder of the optical fiber, which did not receive any specific processing, conveys the light without being visible on the surface.

(a)

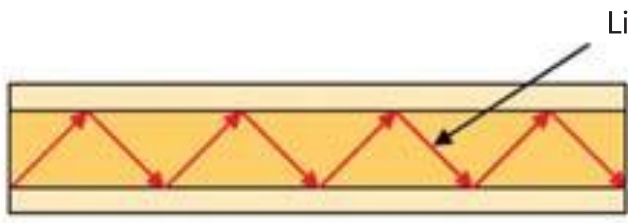

Original optical fiber
Light rays

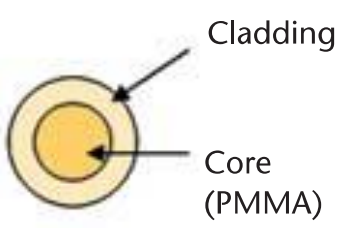

(b)

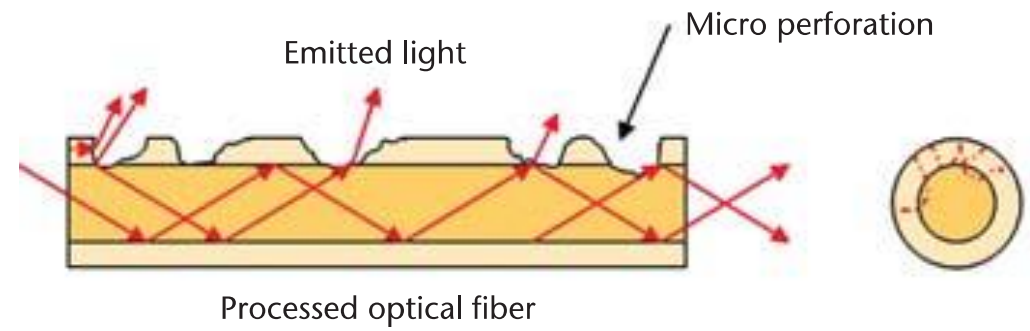

Figure 2: Principle of lateral light emission. a) Original optical fiber, b) processed optical fiber

Two processing techniques have been developed for optical fibers. The first is a mechanical treatment by the projection of micro particles with different velocities on the optical fiber's cladding. The result is presented in Fig. 3. The second technique uses different chemical solvents to make these micro perforations; this method seems to produce a better final result. (A chemically processed cladding surface is shown in Fig. 4). Finally, Fig. 5 shows the chemically processed fiber obtained by a scanning electron microscope.

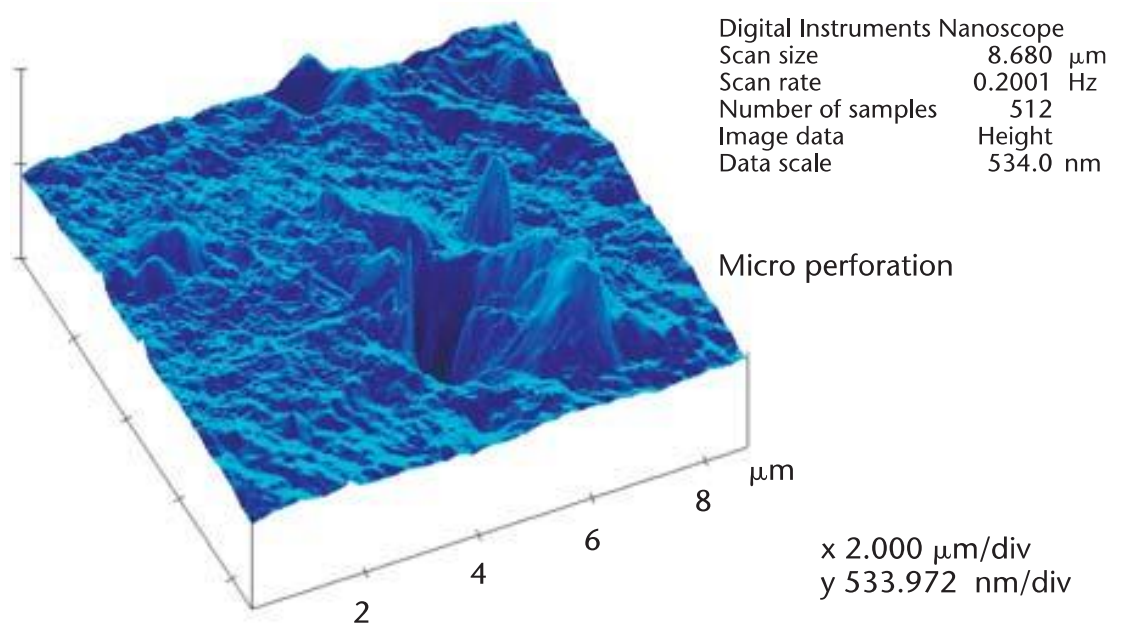

Figure 3: Micro perforation obtained by mechanical treatment (particle projected on the cladding of optical fiber). Picture obtained by nanoscope 


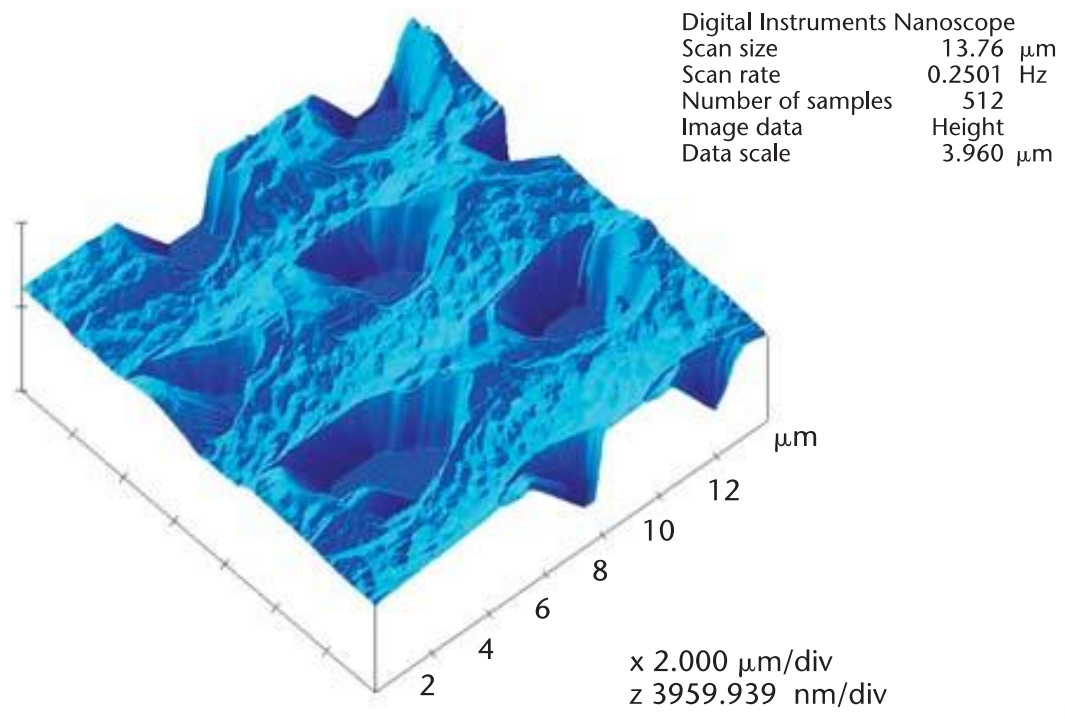

Figure 4: Micro perforation obtained by chemical treatment (solvent action on the cladding of optical fiber). Picture obtained by nanoscope

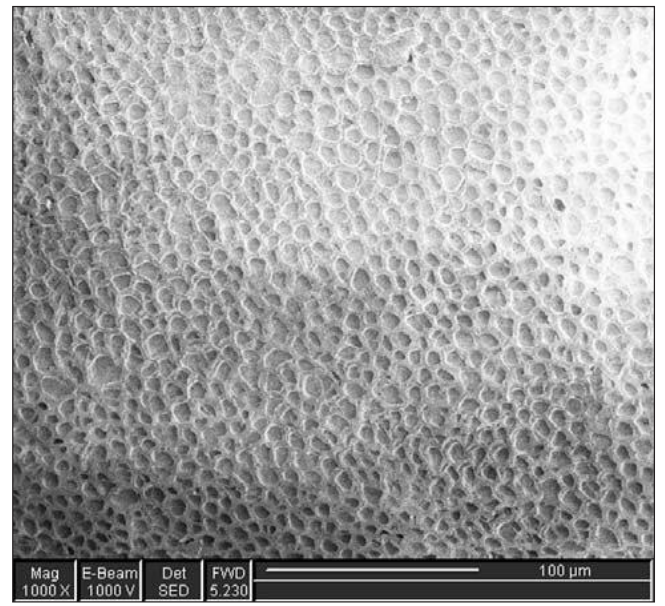

Figure 5: Micro perforation obtained by chemical treatment (solvent action on the cladding of optical fiber). Picture obtained by scanning electron microscope

There are three methods that are used to light ON and OFF static patterns on the fabric (texts, logos and scanned pictures), which we adapted to develop our own technique. A basic fabric is used in the first method. The lighting zone to be processed, which is composed of optical fibers, is delimited by a stencil key. The picture remains static - with eventual color changes-but can offer quite a high resolution.

In the second method, the zone to be lit is formed during weaving on a Jacquard loom before being processed. The remaining, inactive fabric is composed of the floating fibers on the back of the fabric.

A third method uses a two-layer adapted basic-velour fabric that makes optical fibers as visible as possible, but with sufficient consistency of fabric structure. Prior to the weaving process, the optical fibers are chemically treated, enabling the specific dynamic lighting zones to be created.

We modified these techniques by creating specific weaving armor and an adapted lighting control in order to generate variable information on the same fabric zone. We developed a matrix that makes it possible to display a great deal of basic information, such as texts, logos or other patterns, in a static or dynamic way. Because a fabric display can only be produced by columns made of a single optical fiber or group of fibers, we had to create lines artificially. Similar to the process that would be used with two superimposed patterns to be lightened on the same column, this involves alternating two consecutive weft fibers - one for the first pattern, and the other for the second. Each is processed on a precise section in order to re-emit light at a specific place. 
The principle is the same for three superimposed patterns, except that one fiber is taken out of three for each pattern. When the weaving is sufficiently tight, a visual impression is given of full, enlightened zones. Warp yarns will be able to help diffuse the light toward the dark zones between lightened segments. The number of rows to be produced seems limited by the technique, insofar as, on the same unit zone, more dark zones are produced than lightened ones. The appreciation of the definition will then be based on the size of the pixels and the screen, in addition to the distance from which people watch the screen.

Various light sources can be used to feed the matrix. The choice mainly depends on the number of fibers connected to each source and the level of power consumption. For the first prototypes, we used high luminous LEDs that are $3 \mathrm{~mm}$ in diameter. LED technology has many advantages, as diodes can be easily driven by electronics under low voltages ( $2 \mathrm{~V}$ to $4 \mathrm{~V}$, depending on the color). Therefore, many "light effects" can be generated on the display, such as flashing or varying the intensity of the light, providing all kinds of animated movies.

The very first OFFD, has been displayed on a jacket. It comprises a screen matrix specially designed to display on one line three $60 \mathrm{~mm} \times 60 \mathrm{~mm}$ alphanumeric characters, each made up of three rows and three columns using $0.5 \mathrm{~mm}$ diameter optical fibers and a 7 fibers $/ \mathrm{cm}$ width density. Each pixel is composed of four fiber segments and is controlled by one LED located in the lining of the cloth, on one side of the OFFD. The color of the pixels is determined by the corresponding LEDs.

OFFDs offer another possibility: Although the definition is limited by the number of rows, it is possible to repeat on fabric the same line of characters or patterns in the direction imposed by optical fibers. The fixed or animated pattern reproduction can be used for purely decorative applications; for example, to create a mural tapestry adapting its colors to the clothes worn by the occupants of a room.

Optical fiber screens provide access to simple and animated visual information, such as texts or pictograms. It is possible to download, create or exchange visuals via the appropriate Internet gateway. Conceivably, images or text could be sent using wireless technology from a computer or a mobile Internet terminal to an article of clothing.

The main functions of the new prototypes are:

- To "be seen," for security, publicity, recreational or aesthetic purposes

- To show one's affiliation or support for a group

- To personalize one's clothing according to the latest fashions

- To communicate or exchange information or to signpost advice.

Fabrics based on flexible display technology have the obvious potential to influence fashion designers, but they have a variety of other useful applications as well. OFFDs can be used as displays for mobile phones, PDAs (personal digital assistants), wearable computers and other portable electronic devices.

There is also enormous potential for firefighting and police applications. For example, information and warnings could be displayed on clothes-which could both increase public safety and help officers and firefighters to operate in remote and challenging conditions.

The interior of cars contains many flexible elements that could be used to display relevant information that might help drivers navigate or avoid accidents. Finally, houses and buildings could use OFFD technology to display or enhance drawings, pictures and lighting.

In this digital age, information is virtually everywhere and a multitude of screen and display technologies will be necessary to keep up with the demand. OFFDs have shown great promise as a new and interesting way to present images and information.

\subsection{Passive textile displays based on electrochromical compounds}

Materials that can undergo a reversible color change, upon the application of an external stimulus, are technically known as chromic materials. Research into the application of such species has been extensive due to the ability of color change to effectively convey important information, i.e. traffic lights, advertisement billboards etc. (Mortimer et al, 2006; Somani et al, 2003; Tehrani et al, 2006). Materials that display chromic properties are classified by the type of stimuli that induces their color change. For example, photochromics and thermochromics see a change in color when there is a change in light, or a change in heat, respectively. Both thermo- and photochromic materials have current applications within textile fields. Photochromics, when applied to textiles have been used in solar protection through the monitoring and notification of UV radiation levels (Shanmugasundaram, 2008). In addition, for added interest within fashion and interior furnishings, wallpapers treated with thermochromics, when heated by nearby 
radiators, 'grow' flower buds across a vine (Sierra, 2008). Photochromic products include pillow covers which reveal a motif when exposed to sunlight (Zainzinger, 2009). When the heat or sunlight is removed, the flowers and motif disappears correspondingly.

On the other hand, an electrochromic compound is one in which a reversible change in color occurs when an electrical voltage is applied. Many materials express electrochromic properties, including metal oxides, the Prussian family, rare earth phthalocyanines and conducting polymer systems. Products that harness the fascinating spectroelectrochemical properties of electrochromic materials, currently available on the market, are predominantly rigid in their form and include the glass windows of buildings and cars which can darken reversibly (Sage Electrochromics, 2012; Gentex Corporation, 2012).Other proposed applications include re-usable price labels, camouflage materials and controllable light reflective or lighttransmissive displays for optical information and storage (Mortimer et al, 2006; Rosseinsky et al, 2001; Silver et al, 1989).

The traditional structure of an electrochromic device (ECD) is that of a seven-layer electrochemical cell with a rigid sandwich structure (Figure 6).

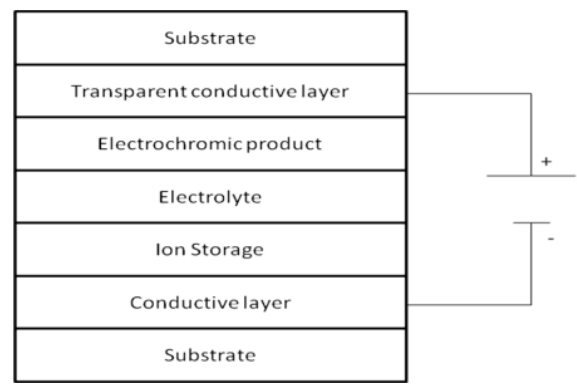

Figure 6: A traditional ECD with a seven-layer sandwich structure

An electrochromic material is coupled to both a suitable solid or liquid electrolyte (ionic conductor) and an ionic storage layer. These three layers are sandwiched between two conductors (electrodes), with at least one of these, also requiring transparency. These are then held between two protective substrates, typically transparent glass, completing the device. A number of academic and commercial research groups have been working on the development of ECDs that are flexible (Andersson et al, 2007; White et al, 2009; Coleman et al, 1999; Ma et al, 2008). For example, Mecerreyes and co-workers have published a flexible all-polymer ECD, using a plastic substrate (Mecerreyes et al, 2004). Poly(3,4-ethylenedioxythiophene), PEDOT, has been utilized, and acts simultaneously as both the electrode and the electrochromic material. The transparent conducting layer of the classical configuration is therefore eliminated, resulting in a device requiring only five-layers. However, flexible textiles treated with electrochromics are not readily available. Therefore, the combination of chromic materials with flexible textiles grants a new opportunity to create communicative flexible displays for clothing, interior furnishings or flags. The change in color could be used for protection and safety applications or for adding further interest or fashion to an item.

Five generations of EC displays have been realized since last ten years in GEMTEX laboratory. First, second and third generations use PET sheets with ITO transparent conductive layer (Figures 7-9), textile substrate containing an electrochromic compound is placed between them. Therefore, the hand is more plastic than textile. Fourth and fifth generations (Figures 10 and 11) are fully textile based without PET ITO coated sheets. The fourth generation is based on 3 layers' textile functionalized fabrics and the fifth generation is made of only one-layer textile substrate that is locally functionalized.

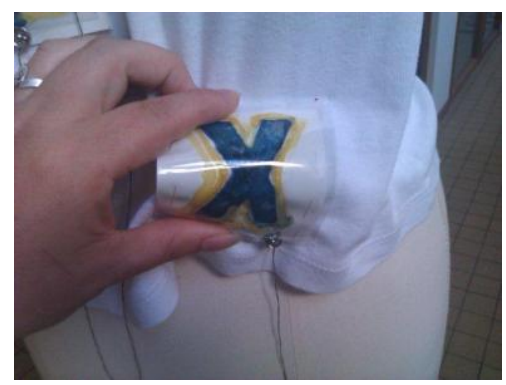

Figure 7: First generation (1G) of GEMTEX EC displays 

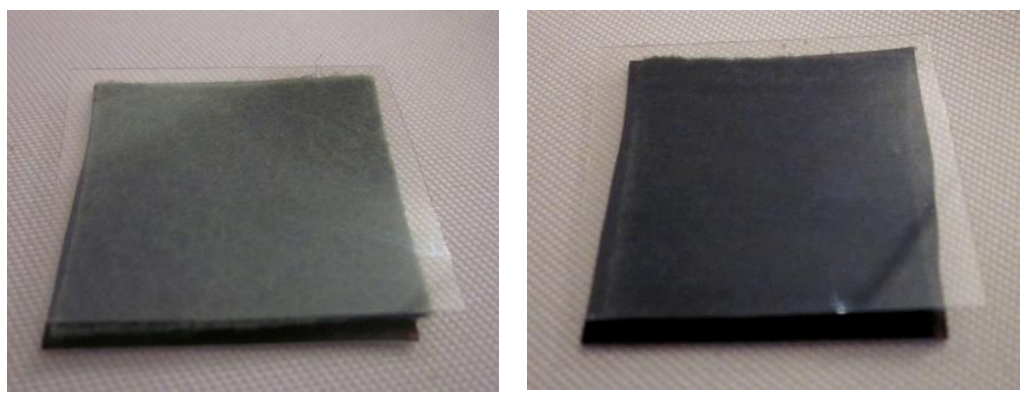

Figure 8: Second generation (2G) of GEMTEX EC displays
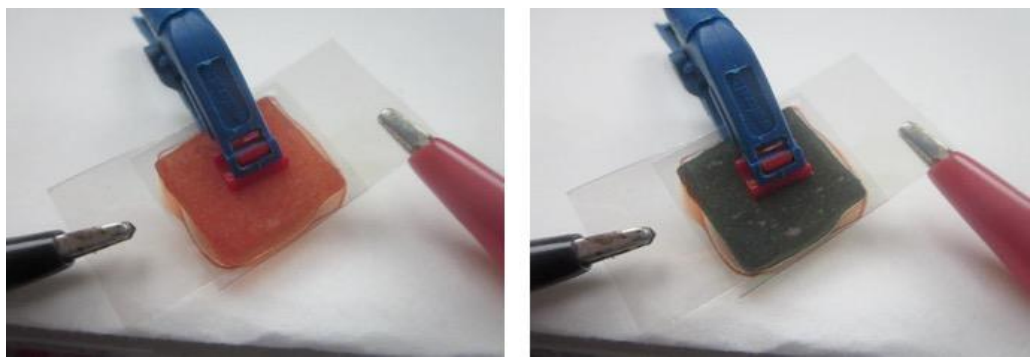

Figure 9: Third generation (3G) of GEMTEX EC displays

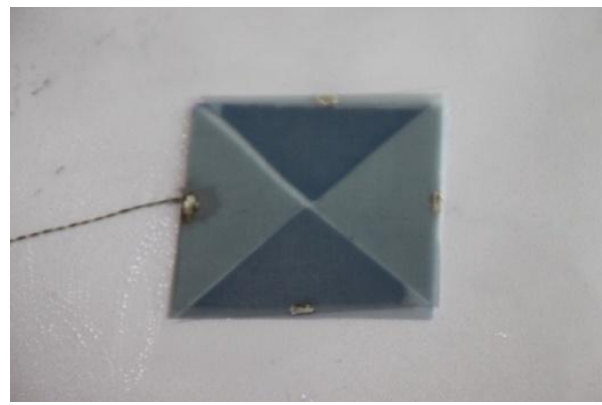

Figure 10: Fourth generation (4G) of GEMTEX EC displays

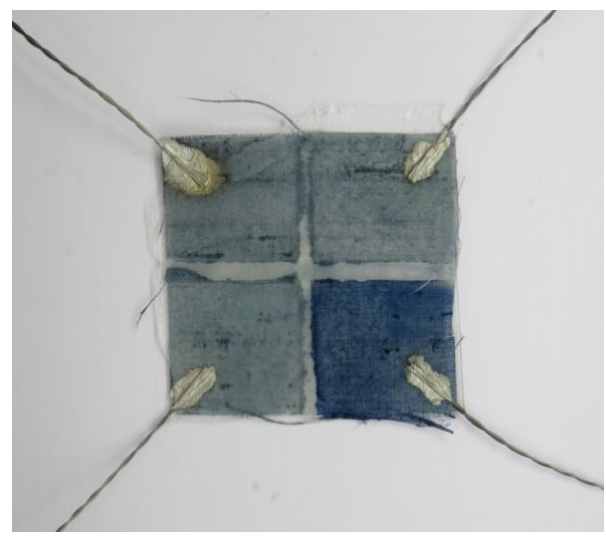

Figure 11: Fifth generation (5G) of GEMTEX EC displays (Moretti et al, n.d.)

\section{CONCLUSION}

New approaches towards the design and development of textile based flexible displays able to emit or reflect light should help designers to enhance their imagination and realize new exciting products (Koncar, 2005; Meunier et al, 2011; Kelly et al, 2013a; Kelly et al, 2013b; Moretti et al, 2014). Different areas are concerned such as fashion design, home textiles, technical textiles, car interior etc. The main issue that will 
have to be addressed concerning optical fibers based displays is related to their cost and integration. Electrochromic displays on the other side should be improved in order to extend their life time and make them more reliable.

\section{REFERENCES}

[1] Andersson, P., Forchheimer, R., Tehrani, P., Berggren, M.: "Printable All-Organic Electrochromic Active-Matrix Displays", Advanced Functional Materials, 17 (16), 3074-3082, 2007. doi: 10.1002/adfm.200601241

[2] Coleman, J.P., Lynch, A.T., Madhukar, P., Wagenknecht, J. H.: "Printed, flexible electrochromic displays using interdigitated electrodes", Solar Energy Materials and Solar Cells, 56 (3-4), 395-418, 1999. doi: 10.1016/S0927-0248(98)00144-5

[3] Gentex Corporation - A Smarter Vision: "Automotive Products" URL http://www.gentex.com/automotive/product-categories (last requested: 2012-08-15)

[4] J. Silver. "Chemical chameleons for electronics," New Scientist, no. 1684, pp. 49-51, Sept. 1989.

[5] Kelly, F.M., Meunier, L., Cochrane, C., Koncar, V.: "Polyaniline: Application as Solid State Electrochromic in a Flexible Textile Display", Displays, 34 (1), 1-7, 2013. doi: 10.1016/j.displa.2012.10.001

[6] Kelly, F.M., Cochrane, C., Koncar, V.: "Evaluation of Solid or Liquid Phase Conducting Polymers within a Flexible Textile Electrochromic Device" IEEE/OSA Journal of Display Technology, 9 (8), 626 - 631, 2013. doi: 10.1109/JDT.2013.2255581

[7] Koncar, V.: "Optical fiber fabric display - OFFD", Optics \& Photonics News, 16 (4), 40-44, 2005. doi: 10.1364/OPN.16.4.000040

[8] Ma, C., Taya, M., Xu, C.: "Flexible electrochromic device based on poly (3,4-(2,2dimethylpropylenedioxy)thiophene)", Electrochimica Acta, 54 (2), 598-605, 2008. doi: 10.1016/j.electacta.2008.07.049

[9] Mecerreyes, D., Marcilla, R., Ochoteco, E., Grande, H., Pomposo, J. A., Vergaz, R., Sánchez Pena, J. M. :"A simplified all-polymer flexible electrochromic device", Electrochimica Acta, 49 (21), 35553559, 2004. doi: 10.1016/j.electacta.2004.03.032

[10] Meunier, L., Kelly, F.M., Cochrane, C., Koncar, V.: "Flexible displays for smart clothing: Part II Electrochromic displays", Indian Journal of Fibre \& Textile Research, 36 (4), 429-435, 2011.

[11] Moretti, C., Tao, X., Koncar, V., Koehl, L.: "A Study on Electrical Performances and Lifetime of a Flexible Electrochromic Textile Device", Autex Research Journal, 14 (1), 2014. doi: 10.2478/aut-2014-0003

[12] Moretti, C., Koncar, V.: Patent pending - FR 1770589, "Structure électro chromique comportant une couche support déformable", ENSAIT

[13] Mortimer, R.J., Dyer, A. L. , Reynolds, J. R.: "Electrochromic organic and polymeric materials for display applications”, Displays, vol. 27 (1), 2-18, 2006. doi: 10.1016/j.displa.2005.03.003

[14] Rosseinsky, D.R, Mortimer, R. J.: "Electrochromic Systems and the Prospects for Devices," Advanced Materials, 13 (11), 783-793, 2001. doi: 10.1002/1521-4095(200106)13:11<783::AIDADMA783>3.0.CO;2-D

[15] Sage Electrochromics. Inc.: "Sage Glass Technology" URL http://www.sage-ec.com/ (last requested: 2012-08-12)

[16] Shanmugasundaram, O. L.: "Smart and Intelligent Textiles", URL http://www.indiantextilejournal.com/articles/FAdetails.asp?id=852. (last requested: 2018-10-17)

[17] Sierra, M.B.: "Thermochromic", URL http://www.techpin.com/thermochromic/ (last requested: 2008-05-26)

[18] Somani, P.R., Radhakrishnan, S.: "Electrochromic materials and devices: present and future", Materials Chemistry and Physics, 77 (1), 117-133, 2003. doi: 10.1016/S0254-0584(01)00575-2

[19] Tehrani, P., Isaksson, J., Mammo, W., Andersson, M. R., Robinson, N. D., Berggren, M.: "Evaluation of active materials designed for use in printable electrochromic polymer displays", Thin Solid Films, 515 (4), 2485-2492, 2006. doi: 10.1016/j.tsf.2006.07.149

[20] White, C.M., Gillaspie, D. T., Whitney, E., Lee, S.H., Dillon, A. C.: "Flexible electrochromic devices based on crystalline WO3 nanostructures produced with hot-wire chemical vapor deposition", Thin Solid Films, 517 (12), 3596-3599, 2009. doi: 10.1016/j.tsf.2009.01.033

[21] Zainzinger, E. (2009, Nov.). Fashionable color changing flu-masks [Webpage]. Available: http://www.talk2myshirt.com/blog/archives/3113 (last requested: 2009-11-03) 


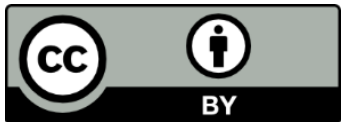

(C) 2018 Authors. Published by the University of Novi Sad, Faculty of Technical Sciences, Department of Graphic Engineering and Design. This article is an open access article distributed under the terms and conditions of the Creative Commons Attribution license 3.0 Serbia (http://creativecommons.org/licenses/by/3.0/rs/). 